\title{
Development of Machine Learning Models for Gas Identification Based on Transfer Functions
}

\author{
G. Imamura ${ }^{1}$, G. Yoshikawa ${ }^{1,2}$, T. Washio ${ }^{3}$ \\ ${ }^{1}$ World Premier International Research Center Initiative (WPI), \\ International Center for Materials Nanoarchitectonics (MANA), \\ National Institute for Materials Science (NIMS), Tsukuba, Ibaraki, 305-0044, Japan \\ ${ }^{2}$ Materials Science and Engineering, Graduate School of Pure and Applied Science, \\ University of Tsukuba, Tennodai 1-1-1 Tsukuba, Ibaraki 305-8571, Japan \\ ${ }^{3}$ The Institute of Scientific and Industrial Research, Osaka University, \\ Mihogaoka 8-1, Ibaraki, Osaka 567-0047, Japan
}

\begin{abstract}
:
Potential applications using gas sensors are expected in various fields by combining MEMS sensors with advanced Information and Communication Technology. In a conventional gas identification approach, gas injection patterns must be strictly controlled to obtain a comparable sensing features which are used for gas identification. Toward a simpler gas sensing system than existing ones, a gas identification protocol which does not depend on gas injection patterns is required. In this study, we have developed a data processing method for gas identification by integrating system identification and machine learning. By using transfer functions as a feature descriptor, we have developed gas identification models which can identify gas species with an arbitrary gas input pattern. For solvent vapors, we have successfully identified gas species with an accuracy of $0.98 \pm 0.03$ even with a random gas injection pattern. We have also identified complex samples, that is odors of herbs and spices, with an accuracy of $0.94 \pm 0.04$, showing the high feasibility of the present approach.
\end{abstract}

Key words: gas identification, machine learning, system identification, transfer function, membranetype surface stress sensors.

\section{Introduction}

Gas sensors have been attracting much attention owing to their potential applications as Internet of Things (loT) devices. To achieve advanced applications of gas sensors including artificial olfaction, it is necessary to establish data processing protocols which can effectively identify gas species from the sensing signals. In a conventional gas identification approach, gas injection pattern must be strictly controlled for every measurement in order to identify gas species from sensing signals; otherwise the measurement data cannot be compared with each other. Toward a simple measurement system, a data processing method which enables gas identification with an arbitrary gas injection pattern is highly required.

In this study, we have developed a new gas identification protocol based on system identification and machine learning. We employed a transfer function-the relationship between the gas input pattern and the output sensing signals as a feature descriptor for machine learning.

\section{Transfer Functions}

A transfer function is a mathematical representation to describe a process model of a system. For a gas sensing system in which an output sensing signal $y(t)$ is linear in the gas input pattern $x(t), y(t)$ can be described as a following form:

$y(t)=\int_{0}^{t} h_{g}(\tau) x(t-\tau) d \tau$

where $h_{g}(t)$ is the time-domain transfer function, which is determined by the interaction between the sensor and a gas $g$. As $h_{g}(t)$ is independent of $\mathrm{x}(\mathrm{t})$, gas species can be identified by focusing on $h_{g}(t)$. Applying the Fourier transform on Equation (1) yields the frequency-domain expression:

$Y(f)=H_{g}(f) X(f)$ 
where $X(f), Y(f), H_{g}(f)$ are the frequency-domain expressions for the gas input pattern, the output sensing signal, and the transfer function, respectively.

\section{Experimental}

Figure 1a shows the schematic illustration of the measurement system. Sample gases were diluted and injected to the chamber where a sensor chip is set by controlling the two mass flow controllers (MFCs): MFC1 and MFC2. The gas flow rate of MFC1 varied from 0 to 100 sccm according to the uniform-random pattern. MFC2 adjusted its gas flow rate so that the total gas flow rate becomes $100 \mathrm{sccm}$. The headspace gases of the following solvents are used as samples: ethanol, toluene, hexane, and water. For a chemical sensor, we utilized a membrane-type surface stress sensor (MSS) ${ }^{[1]}$. We used a four-channel MSS chip, which were coated with four different polymers: poly(vinylidene fluoride), polysulfone, poly(4methylstyrene), and polycaprolactone (Fig. 1b). 30 measurement datasets were obtained for each sample. The measurement time was set at 20 seconds. From the measurement data, $\mathrm{H}_{\mathrm{g}}(\mathrm{f})$ was calculated for each channel. The frequency components blow $1 \mathrm{~Hz}$ were used for analysis.

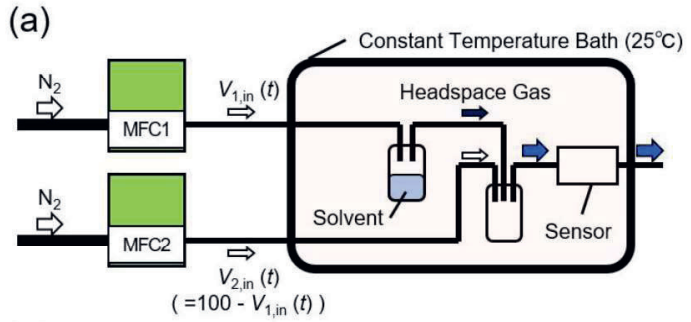

(b)

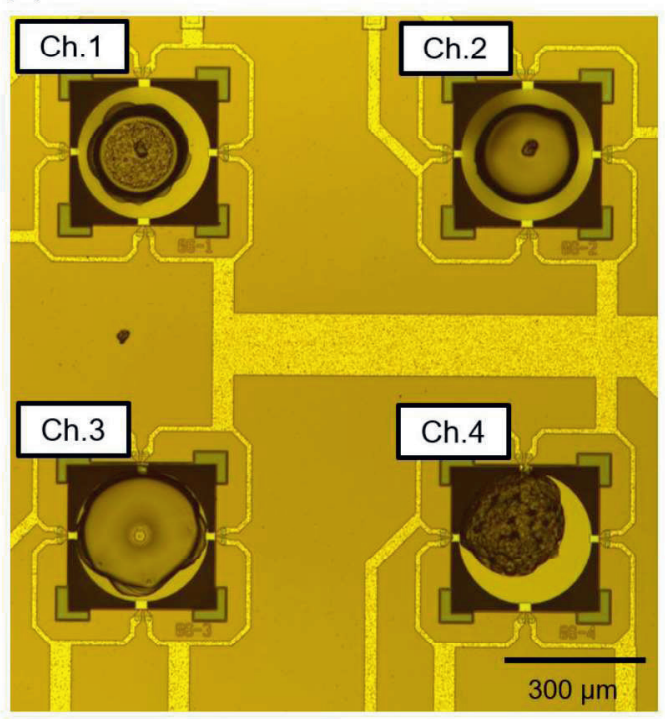

Fig. 1. (a) The schematic illustration of the experimental setup. (b) Optical microscope image of the MSS sensor chip.

\section{Results and Discussion}

On the datasets of concatenated $\mathrm{H}_{g}(\mathrm{f})$, first we performed linear discriminant analysis (LDA). Figure 2 shows the result. It is clear from Fig. 2 that the samples are separated from each other on the scatter plot, reflecting the different transfer functions. Such differences in transfer functions come from the different interactions between the gas species and the receptor materials, that is gas sorption/desorption dynamics in the receptor materials.

We then developed machine learning models which can identify gas species. The optimization of the hyperparameters (i.e. regularization parameter) and the evaluation of the models were performed through the nested cross validation (CV). By using logistic regression (LR) and a support vector machine (SVM) as classifiers, we developed gas identification models, resulting in the classification accuracy of $0.94 \pm 0.04$ and $0.98 \pm 0.03$, respectively.

This gas identification approach using transfer function as a feature descriptor for machine learning can be also adopted to practical samples. We used four herbs and spices (i.e. Cinnamon, Spearmint, Rosemary, and Thyme) as samples and measured their odors with the same experimental setup as shown in Fig.1a. ${ }^{2]}$ Even with a random gas injection pattern, we successfully identify the odors with a classification accuracy of $0.94 \pm 0.04$

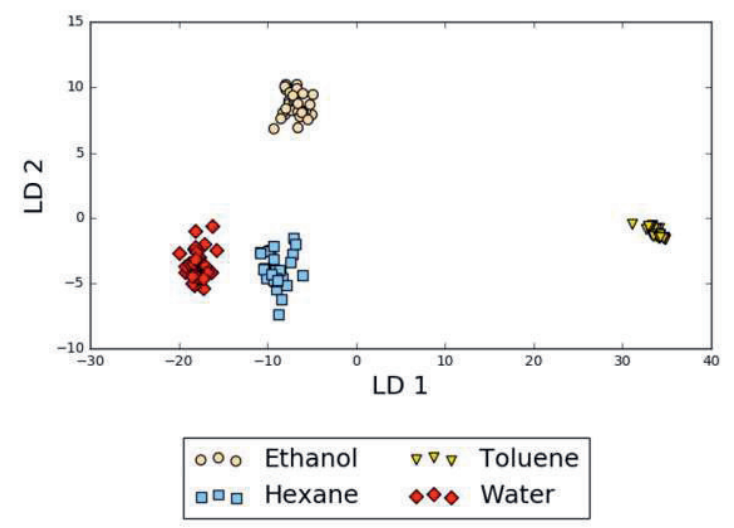

Fig. 2. Scatter plot of the LDA scores.

\section{References}

[1] G. Yoshikawa, T. Akiyama, S. Gautsch, P. Vettiger, H. Rohrer, Nanomechanical Membranetype Surface Stress Sensor, Nano Letters 11, 1044-1048 (2011); doi: 10.1021/nl103901a

[2] G. Imamura, K. Shiba, G. Yoshikawa, Smell identification of spices using nanomechanical membrane-type surface stress sensors, Japanese Journal of Applied Physics 55, 1102B3 (2016); doi: 10.7567/JJAP.55.1102B3 